\title{
Cryptography Triple Data Encryption Standard (3DES) for Digital Image Security
}

\author{
Christy Atika Sari'1,Eko Hari Rachmawanto², Christanto Antonius \\ Haryanto $^{3}$
}

${ }^{1,2,3}$ Informatic Engineering Departement, Computer Science Faculty, Universitas Dian Nuswantoro Email: ${ }^{1}$ atika.sari@dsn.dinus.ac.id, ${ }^{2}$ eko.hari@dsn.dinus.ac.id, ${ }^{3}$ christantoantonius@ gmail.com

\begin{abstract}
Advances in technology in the field of internet, making the Internet become the most popular data transmission media for now. This is certainly not free from the incidence of cyber crime, such as theft and data modification. Given the losses caused by data manipulation is very detrimental to the owner, then the original data can be misused in the cyber world. Cryptography offers an algorithm for randomizing data, so it can not be read by unauthorized people. The cryptography technique was implemented using Triple Data Encyption Standard (3DES) given the results of a randomized cryptographic algorithm, it is possible to arouse suspicion from the viewer. For that will be done the process of insertion of cryptographic files into another media in the form of images commonly referred to as steganography. The steganography technique that will be used is End of File (EOF). The combination of 3DES and EOF in the 64x64 pixel image with grayscale color format produces the fastest image processing time of 173.00192 seconds with the highest Peak Signal to Noise Ratio (PSNR) value of $25.0004 \mathrm{~dB}$, while in the $128 \times 128$ pixel image with gray scale format has produced the highest PSNR $21.0084 \mathrm{~dB}$.
\end{abstract}

Keywords: Cry ptography, Triple Data Encryption Standard, End of File, Peak Signal to Noise Ratio

\section{INTRODUCTION}

Improved technology in the field of internet trigger the occurrence of problems, especially in the field of Internet security. Given the number of Internet media users are quite high, it is possible the occurrence of Internet crimes that occur in the process of sending data through the internet media. Among these are file modifications, or the theft of important data transmitted over the internet. For solving cryptography offers a way to scramble an existing file by using a specific key known only to the user. The technique of cryptography that will be implemented in this research is 3DES. 3DES has a good level of security when compared to other cryptographic techniques [1]. However, cryptography has a weakness, the resulting file looks random, and of course this can cause suspicion for people who see it [2]. For overcoming these deficiencies need to be done the process of insertion of the encrypted file into another media, which is often referred to as steganography. 
The technique of steganography used is EOF. The EOF technique is chosen because it has a fast time [3] compared to other steganographic techniques.

The combination of both methods will be implemented on a 64x64 pixel digital image with a grayscale color format. As for the insertion media that will be used on the method EOF in the form of image 'pepper.png' with grayscale color format measuring $512 \times 512$ pixels. Based on the above problem, the purpose of this research is the implementation of 3DES cryptography algorithm and EOF steganography to secure image data sent via internet media.

\section{METHODS}

\subsection{Cryptography}

Cryptography has actually existed since 4000 years ago in Egypt, which we often know with hieroglyph. In Ancient Rome it was told that Julius Caesar wanted to send a message to someone on the battlefield, but he did not want the message to be known to others [4]. Therefore, Julius Caesar created a way to hide the message, from the problem then created the algorithm Caesar Cipher. In cryptography there are 2 types of encryption models are:

a) Symmetrical: Where in the process of encryption or decryption will use the same key.

b) Asymmetric: Encryption and decryption process using different keys.

The cryptographic algorithm consists of three basic functions, namely [5]:

a) Encryption: Is a process of securing data, by converting the original text into code that is not understood based on the existing algorithm.

b) Key: Is the key that will be used to perform the process of encryption and decryption.

c) Decryption: It is the process of recovering the previously encrypted code back into the original text, using algorithms and keys that have been used previously.

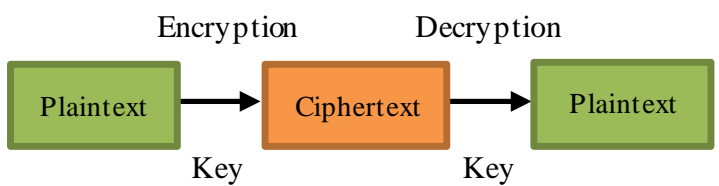

Figure 1. Common cryptography scheme [6]

Based on Figure 1, the encryption process will be performed using plaintext and keys that are processed by a particular algorithm. With this step will be generated a cipher file, which is ready to be sent. For the decryption process, it will be done with the cipher text and the initial key is processed with the initial algorithm.

\subsection{Steganography}

Steganography is a science or art in hiding information by entering the information into other media [3]. So that the existence of the message cannot be known by others. Steganography comes from the Greek Steganós [7] which 
means to hide and Graptos which means writing, so the whole meaning is "writings that are hid". Media commonly used in steganography include:

Image: .bmp, .gif, .jpeg, .jpg, .tiff, .png.

Audio: .wav, .mp3

Text: .rtf, .txt, .pdf, .doc, .docx.

The purpose of steganography is to conceal the existence of a data, by hiding it into a particular media.

\subsection{Triple Data Encryption Standard (3DES)}

3DES is a symmetric cryptography algorithm with cipher block type. Symmetric cryptography is a method that will use the same key in the encryption and decryption process. Cipher block is a type of asymmetric cryptography that has a fixed or fixed bit size, which is 64 bits for DES [8]. 3DES is the development of Double DES algorithm previously available to improve DES security. The 3DES algorithm uses three keys in the encryption and decryption process. The key variations in 3DES can be classified into 3 by using the same 1 key, 2 different keys, or 3 different keys to each other. 3DES encryption using 2 or 3 different keys is still considered robust for current usage [9].

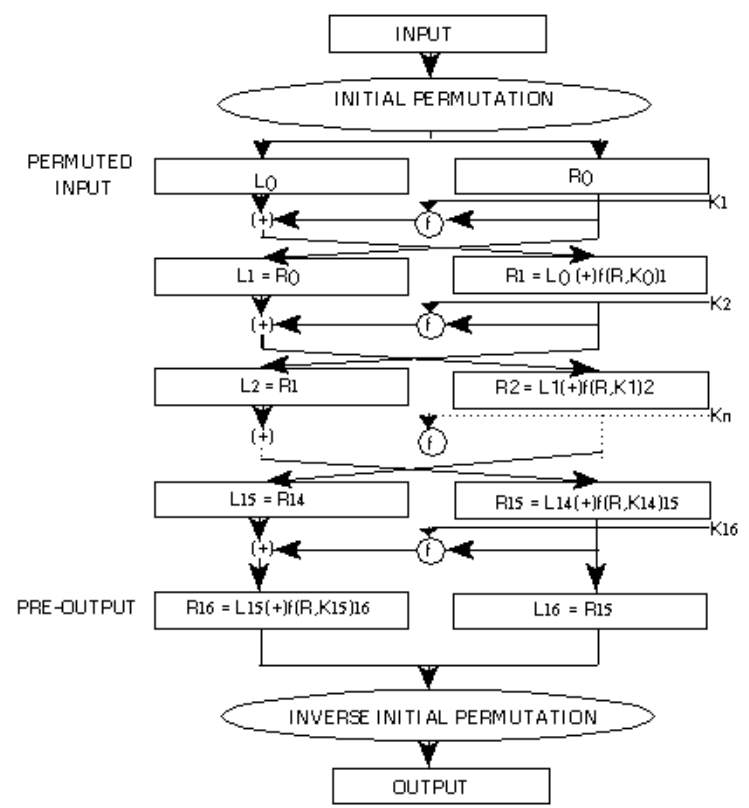

Figure 2. 3DES scheme using permutations

Based on Figure 2 above it can be seen that the sequence of 3DES algorithm scheme. Encryption formulas for 3DES can be described as follows: 
$\mathrm{C}=\mathrm{E} 3_{\mathrm{k} 1, \mathrm{k} 2, \mathrm{k} 3}(\mathrm{P})=\mathrm{E}_{\mathrm{k} 3}\left(\mathrm{D}_{\mathrm{k} 2}\left(\mathrm{E}_{\mathrm{k} 1}(\mathrm{P})\right)\right)$

Where $E 3_{k 1, k 2, k 3}(P)$ is 3 DES encryption for $\mathrm{P}$ using key, are $\mathrm{k}_{1}, \mathrm{k}_{2}$, and $\mathrm{k}_{3}$. Whereas $E_{k n}(P)$ is DES encryption for $\mathrm{P}$ using $\mathrm{k}_{\mathrm{n}}$. For $D_{k n}(P)$ is DES decryption for $\mathrm{P}$ using key $\mathrm{k}_{\mathrm{n}}$. The decryption formula for 3DES can be described as follows:

$P=D 3_{k 1, k 2, k 3}(C)=D_{k 1}\left(E_{k 2}\left(D_{k 3}(C)\right)\right)$

Where $D 3_{k 1, k 2, k 3}(P)$ 3DES decryption of $\mathrm{C}$ using key, are $\mathrm{k}_{1}, \mathrm{k}_{2}$, and $\mathrm{k}_{3}$. Whereas $D_{k n}(P)$ is DES decryption for P using $\mathrm{k}_{\mathrm{n}}$. For $E_{k n}(P)$ is encryption for $\mathrm{P}$ using key $\mathrm{k}_{\mathrm{n}}$

\subsection{Least Significant Bit (LSB)}

The LSB is part of the binary number row that has the least or meaningless value. The location of LSB is the rightmost bit sequence [10]. For example, in a byte 01011001 the LSB bit is the bit located at the far right of "1". Sometimes the letter "b" at the end of the number becomes $01011001 \mathrm{~b}$.

$1 * 2^{7}+1 * 2^{6}+1 * 2^{5}+1 * 2^{4}+1 * 2^{3}+1 * 2^{2}+1 * 2^{1}+1 * 2^{0}=128+64+32+16+8+4+2+1=255$

Figure 3. Example of LSB payload calculation

From the 1st row above, the 1st rightmost digit is 1 , and that is the smallest. The section is called the least significant bit (the least significant bit), while the leftmost part is 128 and is called the most significant bit (Joshi \& Yadav 2015). Least significant bits are often used for the purposes of insertion of data into another digital medium (Gosalia 2016). Suppose the message to be inserted 5 bits $=11010$, then the number of bytes used $=5$ bytes 100101101100100111111001 1000100010100011 (byte used for message insertion) The insertion process message 11010, the insertion result becomes 100101111100100111111000 10001001 10100010, so this LSB method is only replace the first bit.

\subsection{Peak Signal to Noise Ratio (PSNR)}

PSNR is the result of comparison of the maximum value of the image measured by the noise. PSNR in this study will be used to perform the process of comparison of results from images before and after the steganography process. The higher the PSNR value is similar to the image between the comparable images [11]. The formula of the PSNR value can be defined according to equation (3) and equation (4) below.

$P S N R=10 \log _{10}\left(\frac{C^{2} \max }{M S E}\right)$ 
With the value of MSE (Mean Square Error) obtained according to the formula as follows:

$M S E=\frac{1}{M . N} \sum_{X=1}^{M} \sum_{Y=1}^{N}\left(S_{x y}-C_{x y}\right)^{2}$

\subsection{Histogram}

An image histogram is a graph depicting the distribution of pixel intensity values of an image[12], or just a part of an image. In the cipher file his togram if it looks flat for each color intensity is the same and this indicates that the algorithmused cannot provide any guidance for a statistical attack [13] because there is no prominent intensity as in plain file histogram.

\section{RESULTS AND DISCUSSION}

Here, we proposed a scheme both encryption and decryption process as present in Figure 4.
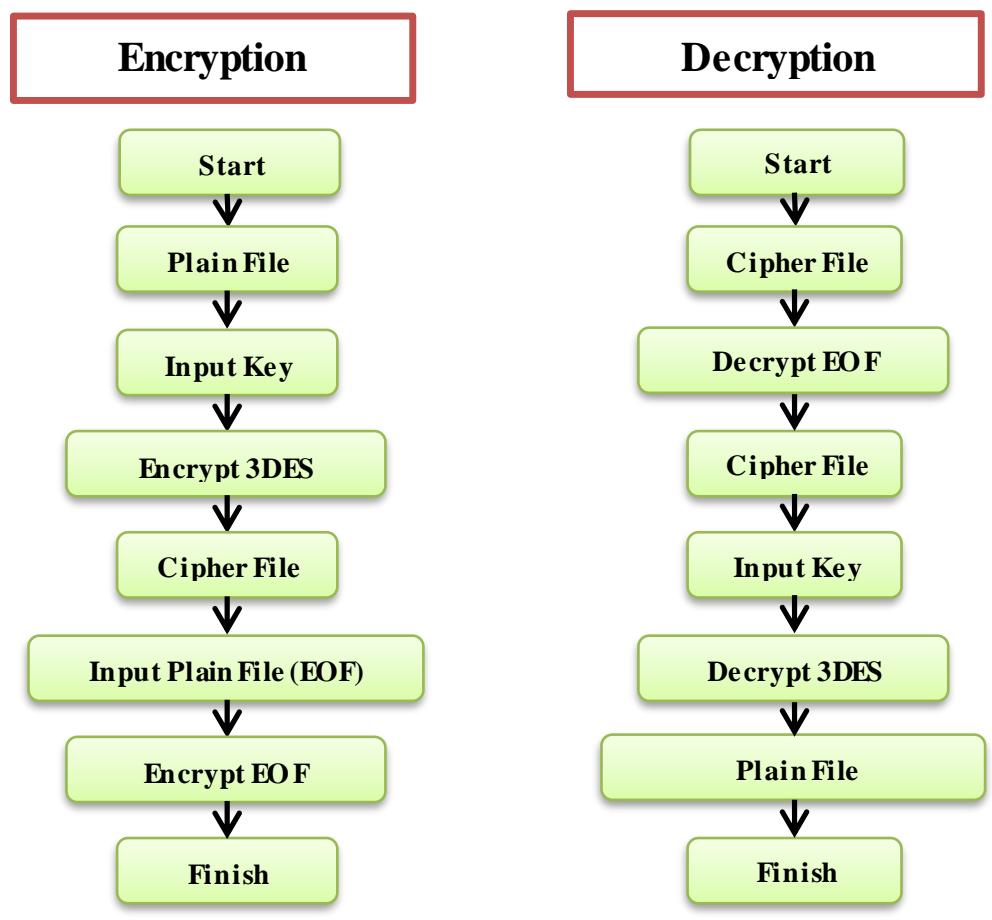

Figure 4. Proposed scheme 3DES-EOF

Based on Figure 4 can be explained the description as follows. For the encryption step, image-shaped data to be tested is first prepared in advance. Input the plain file to be encrypted, then will be asked input in the form of the key as much as 3 pieces of plain text 8 characters that will be inputted to the program to encrypt the 
image with 3DES. Then the cipher file generated from the encryption will be used on the EOF encryption. Previously will be asked for input image that will be used for the process of encryption EOF. After encryption EOF is done will get image file result of cipher file $3 \mathrm{DES}$ and $\mathrm{EOF}$.

For the process of decryption itself, the first requested input in the form of cipher files $3 \mathrm{DES}$ and EOF. Then will be done by using encryption algorithm EOF. After that will be requested input in the form of 3 pieces of key previously used to perform the encryption process of 3DES. After that the 3DES algorithm will run, and generate plain files that can be reused. The image to be used as a sample of this encryption process is the baboon.png that can be seen in Figure 5 with the size of $64 \times 64$ pixels which has the grayscale color format.

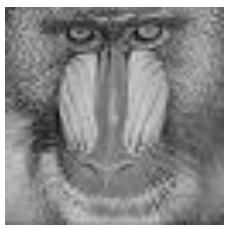

Figure 5. Baboon.png

For the value to be used for the calculation is the first 8 pixels contained in the image baboon.png. The pixels of baboon shown in Figure 6 .

\begin{tabular}{|c|c|c|c|c|c|c|c|c|c|c|}
\hline 1 & gambar $x$ & & & & & & & & & \\
\hline ت & $64 \times 64$ uint8 & & & & & & & & & \\
\hline & 1 & 2 & 3 & 4 & 5 & 6 & 7 & 8 & 9 & 10 \\
\hline 1[ & 80 & 74 & 63 & 114 & 120 & 135 & 94 & 146 & 117 & 110 \\
\hline 2 & 60 & 130 & 130 & 139 & 116 & 120 & 159 & 139 & 124 & 164 \\
\hline 3 & 67 & 70 & 112 & 144 & 141 & 130 & 157 & 148 & 126 & 141 \\
\hline 4 & 74 & 114 & 103 & 123 & 124 & 160 & 121 & 113 & 144 & 126 \\
\hline 5 & 81 & 93 & 133 & 148 & 115 & 153 & 161 & 126 & 170 & 107 \\
\hline 6 & 114 & 85 & 123 & 102 & 138 & 165 & 126 & 164 & 117 & 125 \\
\hline 7 & 96 & 114 & 110 & 142 & 165 & 105 & 130 & 143 & 148 & 123 \\
\hline 8 & 112 & 150 & 124 & 115 & 149 & 169 & 169 & 169 & 147 & 155 \\
\hline 9 & 101 & 117 & 109 & 136 & 106 & 146 & 156 & 149 & 160 & 139 \\
\hline 10 & 120 & 106 & 114 & 145 & 130 & 147 & 158 & 137 & 150 & 137 \\
\hline 11 & 96 & 122 & 126 & 107 & 158 & 117 & 174 & 144 & 137 & 155 \\
\hline 12 & 92 & 114 & 152 & 133 & 137 & 161 & 149 & 162 & 138 & 166 \\
\hline
\end{tabular}

Figure 6. Pixels of baboon.png

From Figure 6 above it can be seen that the pixel value of the first 8 pixels in baboon.png is:

$\begin{array}{llllllll}80 & 74 & 63 & 114 & 120 & 135 & 94 & 146\end{array}$

Once the pixel value is obtained, the pixel will be converted to binary, the binary value to be generated is:

$\begin{array}{rrrr}80 & 74 & 63 & 114 \\ 01010000 & 01001010 & 00111111 & 01110010\end{array}$


After obtaining the binary value of 8 pixels early baboon.png, will be processed into the binary value of the key that will be used in 3DES algorithm. The key to be used consists of 8 characters. The key to be us ed for the 3DES algorithm in this study is 'COMPUTER'. Of the 8 characters will be processed into binary values using ASCII table. The following is the result of the binary key value 'COMPUTER':

$\begin{array}{cccc}\mathrm{C} & \mathrm{O} & \mathrm{M} & \mathrm{P} \\ 01000011 & 01001111 & 01001101 & 01010000 \\ \mathrm{U} & & & \mathrm{R} \\ 01010101 & 01010100 & 01000101 & 01010010\end{array}$

After obtained both the input image and key values, will be the iteration of 3DES calculation. For encryption in plain file, the first step to do is processing by using the initial permutation table in Figure 7, after initial permutation is generated, the value of the existing bit will be split into 2 parts ie $\mathrm{L} 0$ and $\mathrm{R} 0$.

\begin{tabular}{|c|c|c|c|c|c|c|c|}
\hline 58 & 50 & 42 & 34 & 26 & 18 & 10 & 2 \\
\hline 60 & 52 & 44 & 36 & 28 & 20 & 12 & 4 \\
\hline 62 & 54 & 46 & 38 & 30 & 22 & 14 & 6 \\
\hline 64 & 56 & 48 & 40 & 32 & 24 & 16 & 8 \\
\hline 57 & 49 & 41 & 33 & 25 & 17 & 9 & 1 \\
\hline 59 & 51 & 43 & 35 & 27 & 19 & 11 & 3 \\
\hline 61 & 53 & 45 & 37 & 29 & 21 & 13 & 5 \\
\hline 63 & 55 & 47 & 39 & 31 & 23 & 15 & 7 \\
\hline
\end{tabular}

Figure 7. Initial permutation table of DES

As for the encryption on the key, will be done by using the PC-1 to produce CD (k) table. The value of each resulting binary will be split into C0 and D0 which shown in Figure 8.

\begin{tabular}{|c|c|c|c|c|c|c|}
\hline 57 & 49 & 41 & 33 & 25 & 17 & 9 \\
\hline 1 & 58 & 50 & 42 & 34 & 26 & 18 \\
\hline 10 & 2 & 59 & 51 & 43 & 35 & 27 \\
\hline 19 & 11 & 3 & 60 & 52 & 44 & 36 \\
\hline 63 & 55 & 47 & 39 & 31 & 23 & 15 \\
\hline 7 & 62 & 54 & 45 & 38 & 30 & 22 \\
\hline 14 & 6 & 61 & 53 & 45 & 37 & 29 \\
\hline 21 & 13 & 5 & 28 & 20 & 12 & 4 \\
\hline
\end{tabular}

Figure 8. Table PC-1 of DES 
Then the results of C0 and D0 will be processed using the Left Shift table which can be seen in Figure 9.

\begin{tabular}{|l|c|c|c|c|c|c|c|c|c|c|c|c|c|c|c|c|}
\hline Iteration & 1 & 2 & 3 & 4 & 5 & 6 & 7 & 8 & 9 & 10 & 11 & 12 & 13 & 14 & 15 & 16 \\
\hline Left Shift & 1 & 1 & 2 & 2 & 2 & 2 & 2 & 2 & 1 & 2 & 2 & 2 & 2 & 2 & 2 & 1 \\
\hline
\end{tabular}

Figure 9. Left shift iterations

The result of the shift by using Left Shift table will be called C1 and D1, then the result will be processed using PC-2 table as shown below in Figure 10.

\begin{tabular}{|c|c|c|c|c|c|}
\hline 14 & 17 & 11 & 24 & 1 & 5 \\
\hline 3 & 28 & 15 & 6 & 21 & 10 \\
\hline 23 & 19 & 12 & 4 & 26 & 8 \\
\hline 16 & 7 & 27 & 20 & 13 & 2 \\
\hline 41 & 52 & 31 & 37 & 47 & 55 \\
\hline 30 & 40 & 51 & 45 & 33 & 48 \\
\hline 44 & 49 & 39 & 56 & 34 & 53 \\
\hline 46 & 42 & 50 & 36 & 29 & 32 \\
\hline
\end{tabular}

Figure 10. Table PC-2 of DES

At this stage, the key is finished and ready for use in the final calculation process. For the next step, the processing will be done on the plain files that have been processed earlier by using the expansion table. Then the result of the expansion table will be XOR with the result from the PC-2 table as follow in Figure 11.

\begin{tabular}{|c|c|c|c|c|c|}
\hline 32 & 1 & 2 & 3 & 4 & 5 \\
\hline 4 & 5 & 6 & 7 & 8 & 9 \\
\hline 8 & 9 & 10 & 11 & 12 & 13 \\
\hline 12 & 13 & 14 & 15 & 16 & 17 \\
\hline 16 & 17 & 18 & 19 & 20 & 21 \\
\hline 20 & 21 & 22 & 23 & 24 & 25 \\
\hline 24 & 25 & 26 & 27 & 28 & 29 \\
\hline 28 & 29 & 30 & 31 & 32 & 1 \\
\hline
\end{tabular}

Figure 11. Expansion table of DES

After the XOR process will be obtained binary value of 48 bits to be processed using table S-Box and P-Box. This step is a one-time iteration of the 3DES algorithm using the first key. After processing using 3 pieces with 16 different iterations called L16 and R16, the following binary values are obtained bellow and Figure 12. 
0

110

173

$R_{16}: 00000000$

01101110

10101101

01111000

\begin{tabular}{|c|c|c|c|c|c|c|c|c|c|c|}
\hline \multicolumn{11}{|c|}{ 田64x64 uint8 } \\
\hline & & 2 & 3 & 4 & 5 & 6 & 7 & 8 & 9 & 10 \\
\hline 1 & 205 & 190 & 122 & 34 & 0 & 110 & 173 & 120 & 122 & 161 \\
\hline 2 & 209 & 134 & 139 & 11 & 231 & 216 & 103 & 5 & 124 & 137 \\
\hline 3 & 84 & 211 & 177 & 52 & 87 & 214 & 13 & 73 & 243 & 48 \\
\hline 4 & 249 & 219 & 191 & 86 & 61 & 215 & 178 & 67 & 200 & 83 \\
\hline 5 & 69 & 230 & 44 & 149 & 231 & 209 & 111 & 226 & 253 & 105 \\
\hline 6 & 189 & 119 & 18 & 185 & 207 & 173 & 224 & 178 & 170 & 195 \\
\hline 7 & 65 & 28 & 126 & 41 & 210 & 94 & 88 & 72 & 165 & 160 \\
\hline 8 & 218 & 85 & 194 & 164 & 80 & 34 & 2 & 102 & 123 & 217 \\
\hline 9 & 62 & 4 & 133 & 93 & 167 & 45 & 206 & 29 & 217 & 152 \\
\hline 10 & 143 & 213 & 244 & 164 & 48 & 48 & 71 & 220 & 17 & 37 \\
\hline 11 & 84 & 114 & 60 & 78 & 35 & 234 & 195 & 221 & 76 & 71 \\
\hline 12 & 77 & 3 & 48 & 9 & 173 & 189 & 240 & 40 & 169 & 105 \\
\hline 13 & 82 & 248 & 31 & 166 & 227 & 221 & 149 & 139 & 214 & 148 \\
\hline 14 & 237 & 86 & 235 & 75 & 83 & 130 & 239 & 18 & 111 & 190 \\
\hline 15 & 32 & 83 & 65 & 30 & 70 & 104 & 223 & 123 & 74 & 210 \\
\hline
\end{tabular}

Figure 12. A pixels of encryption result

The process of the EOF algorithm has been done by inserting the pixel value at the end in an image used as a medium. Results from the EOF process. The image to be used in the EOF process in this study is the image of 'peppers.png' measuring 512x512 pixels in grayscale color format as shown in Figure 13.

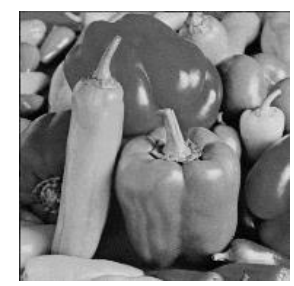

Figure 13. Peppers.png used in EOF process after DES

Based on the Figure 14, can be seen that the value of the results of 3DES calculation has been successfully inserted in the image by using the algorithm EOF. Image data to be used in this study as many as 10 pieces of images taken from petitcolas.net [9] with description, 5 images processed size $64 \times 64$ pixels and $128 \times 128$ pixels. The image to be used has a .png and .bmp format. For the encryption process will be done using 3DES cryptography algorithm and EOF steganography. 


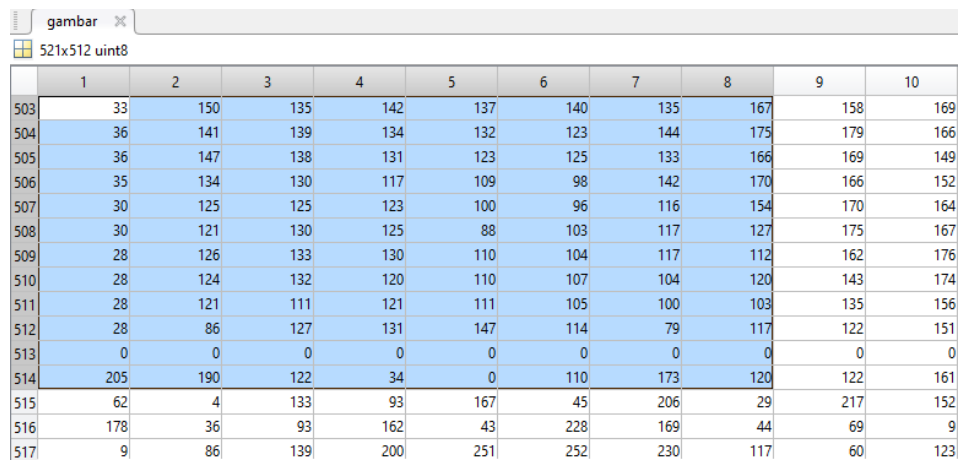

Figure 14. A result of DES-EOF

The time comparison between images with the size of $64 \times 64$ pixels with a $128 x 128$ pixel image shown in Table 1. It appears that the fastest processing time of a $64 \times 64$ pixel image is 172.00139 seconds, while for $128 \times 128$ images it is 680.99123 seconds.

Table 1. Time elapse of 3DES-EOF

\begin{tabular}{ccccc}
\hline \multirow{2}{*}{ No } & \multirow{2}{*}{ Name of Image } & Size of Image & \multicolumn{2}{c}{ Time Elapse (in second) } \\
\cline { 3 - 4 } & & $64 \times 64$ & 179.27272 & 174.29913 \\
\hline 1 & baboon.png & $64 \times 64$ & 176.28819 & 175.29316 \\
2 & bear.bmp & $64 \times 64$ & 174.19215 & 179.29913 \\
3 & f16.png & $64 \times 64$ & 177.99314 & 178.99182 \\
4 & lochness.bmp & $64 \times 64$ & 173.00192 & 172.00139 \\
5 & papermachine.png & $128 \times 128$ & 680.99123 & 683.20013 \\
6 & baboon.png & $128 \times 128$ & 685.00134 & 689.99132 \\
7 & bear.bmp & $128 \times 128$ & 688.11324 & 681.33045 \\
8 & f16.png & $128 \times 128$ & 683.04834 & 686.91344 \\
9 & lochness.bmp & $128 \times 128$ & 689.13775 & 683.22134 \\
10 & papermachine.png & & & \\
\hline
\end{tabular}

The result of 3DES-EOF ont the encryption process shown in Table 2 and Table 3. PNSR value of EOF is shown in Table 4.

Table 2. Result of 3DES-EOF on the encryption process $64 \times 64$ pixels

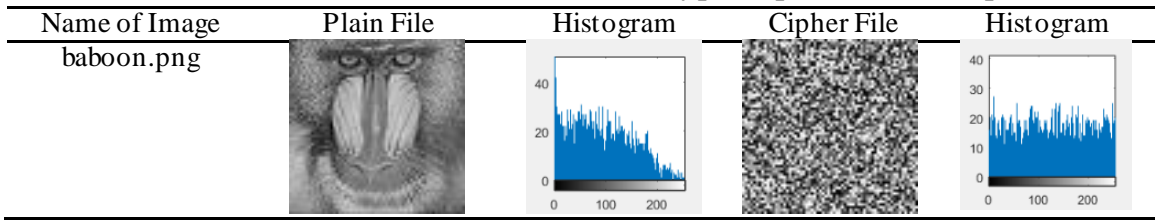




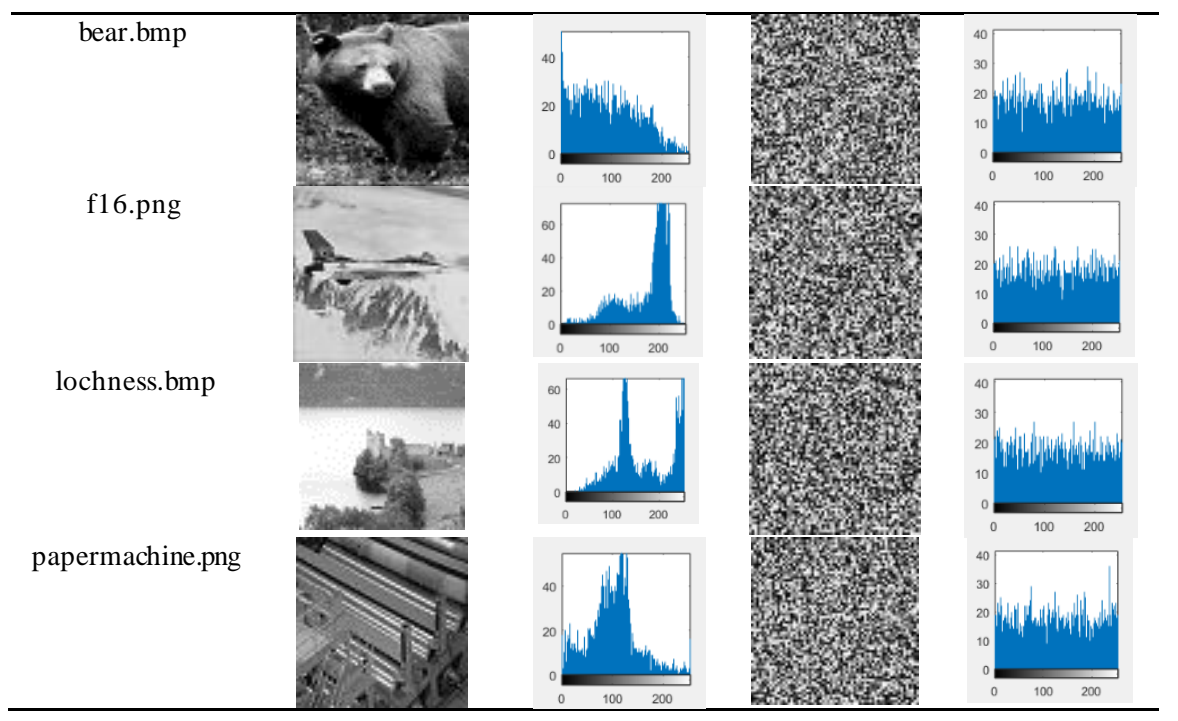

Table 3. Result of 3DES-EOF on the encryption process 128x128 pixels

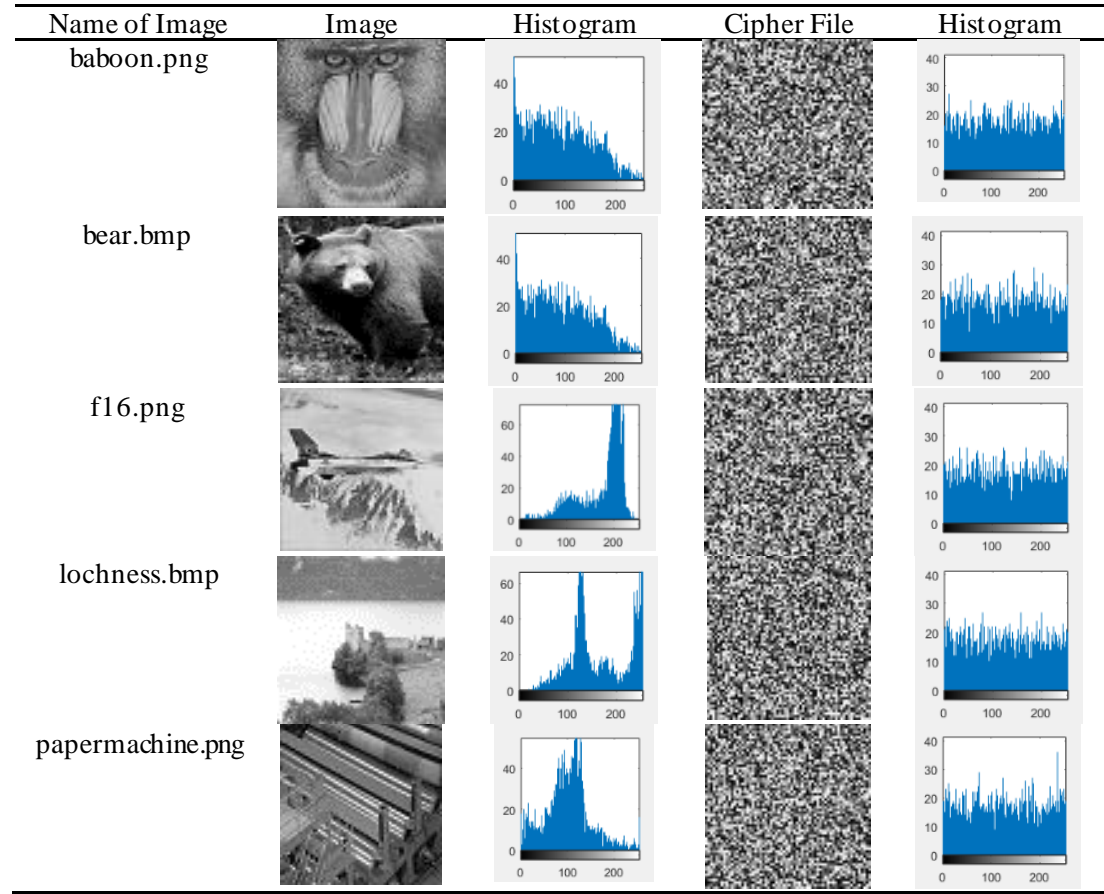


Table 4. PSNR value of EOF

\begin{tabular}{|c|c|c|c|c|c|}
\hline \multirow{2}{*}{ Name of Image } & \multirow{2}{*}{ Plain File } & \multicolumn{2}{|c|}{ EOF 64x64 pixels } & \multicolumn{2}{|c|}{ EOF $128 \times 128$ pixels } \\
\hline & & Stego Image & PSNR (db) & Stego Image & PSNR (db) \\
\hline baboon.png & & & 24.9788 & & 21.0081 \\
\hline bear.bmp & & & 25.0702 & & 20.9198 \\
\hline f16.png & & & 24.9302 & & 20.9793 \\
\hline lochness.bmp & & & 25.0549 & & $21.0301 \mathrm{~dB}$ \\
\hline papermachine.png & & & 25.0004 & & 21.0084 \\
\hline
\end{tabular}

Based on Table 4, it can be seen that the resulting PSNR values vary from $20 \mathrm{~dB}$ to $25 \mathrm{~dB}$ for the tested image. We tested several plain file using peppers.png as shown in Figure 13.

\section{CONCLUSION}

The combination of both algorithms has been successfully applied to grayscale images of $64 \times 64$ pixels and 128x128 pixels with image format .png and .bmp. With the lowest travel time for $64 \times 64$ pixel images is 172.00139 seconds and 680.99123 seconds for images with $128 \times 128$ pixels in size. For the resulting PSNR values vary between $20 \mathrm{~dB}$ to $25 \mathrm{~dB}$. Suggestions that can be given for further research is the application can be implemented with the use of GUI with other image format as well as in color image format.

\section{REFERENCES}

[1] Thakur, J., \& Kumar, N. (2011). DES, AES and Blowfish: Symmetric Key Cryptography Algorithms Simulation Based Performance Analysis. International Journal of Emerging Technology and Advanced Engineering, 1(2), 6-12.

[2] Cheddad, A., Condell, J., Curran, K., \& Mc Kevitt, P. (2010). Digital Image Steganography: Survey and Analysis of Current Methods. Signal processing, 90(3), 727-752.

[3] Cole, E. (2003) Hiding in Plain Sight: Steganography and the Art of. Indianapolis, Indiana, Canada: Wiley Publishing. 
[4] Ardy, R. D., Indriani, O. R., Sari, C. A., \& Rachmawanto, E. H. (2017, November). Digital Image Signature Using Triple Protection Cryptosystem (RSA, Vigenere, and MD5). In Smart Cities, Automation \& Intelligent Computing Systems (ICON-SONICS), 2017 International Conference on (pp. 87-92). IEEE.

[5] Sari, W. S., Rachmawanto, E. H., \& Sari, C. A. (2017). A Good Performance OTP Encryption Image Based on DCT-DWT Steganography. Telkomnika, 15(4), 1987-1995.

[6] Kusuma, E. J., Indriani, O. R., Sari, C. A., \& Rachmawanto, E. H. (2017, November). An Imperceptible LSB Image Hiding on Edge Region Using DES Encryption. In Innovative and Creative Information Technology (ICITech), 2017 International Conference on (pp. 1-6). IEEE.

[7] Ardiansyah, G., Sari, C. A., \& Rachmawanto, E. H. (2017, November). Hybrid Method using 3-DES, DWT and LSB for Secure Image Steganography Algorithm. In Information Technology, Information Systems and Electrical Engineering (ICITISEE), 20172 nd International conferences on (pp. 249-254). IEEE.

[8] Nilesh, D., \& Nagle, M. (2014, January). The New Cryptography Algorithm with High Throughput. In Computer Communication and Informatics (ICCCI), 2014 International Conference on (pp. 1-5). IEEE.

[9] Kaur, A. (2017). A Review on Symmetric Key Cryptography Algorithms. International Journal of Advanced Research in Computer Science, 8(4): 358-362.

[10] Watters, P., Martin, F., \& Stripf, H. S. (2008). Visual Detection of LSBEncoded Natural Image Steganography. World Wide Web Internet And Web Information Systems, 5(1): 24-32.

[11] Almohammad, A., \& Ghinea, G. (2010, July). Stego Image Quality and the Reliability of PSNR. In Image Processing Theory Tools and Applications (IPTA), 2010 2nd International Conference on (pp. 215-220). IEEE.

[12] Rachmawanto, E. H., \& Sari, C. A. (2017, October). A Performance Analysis Stegocrypt Algorithm Based on LSB-AES 128 bit in Various Image Size. In Application for Technology of Information and Communication (iSemantic), 2017 International Seminar on (pp. 16-21). IEEE.

[13] Li, X., Zhang, W., Ou, B., \& Yang, B. (2014, July). A Brief Review on Reversible Data Hiding: Current Techniques and Future Prospects. In Signal and Information Processing (ChinaSIP), 2014 IEEE China Summit \& International Conference on (pp. 426-430). IEEE. 